\title{
Une caractérisation des endomorphismes de Lattès par leur mesure de Green
}

\author{
F. Berteloot et C. Dupont
}

Résumé. We show that the Lattès endomorphisms are the only holomorphic endomorphisms of the complex $k$-dimensional projective space whose measure of maximal entropy is absolutely continuous with respect to the Lebesgue measure. As a consequence, Lattès endomorphisms are also characterized by other extremal properties as the maximality of the Hausdorff dimension of their measure of maximal entropy or the minimality of their Liapounov exponents. Our proof uses a linearization method which is of independant interest and a previous characterization by the regularity of the Green current.

Mathematics Subject Classification (2000). 32H50, 32U40, 37C45.

Keywords. Lattès endomorphisms, linearization, maximal entropy mesaure, Hausdorff dimension, Liapounov exponents.

\section{Introduction et résultats}

Les propriétés dynamiques d'un endomorphisme holomorphe $f$ de degré algébrique $d \geq 2$ sur l'espace projectif complexe $\mathbb{P}^{k}$ se reflètent sur son courant et sa mesure de Green. Le courant de Green, noté $T$, est un $(1,1)$-courant positif fermé, obtenu comme la limite des $(1,1)$-formes $\frac{1}{d^{n}} f^{n *} \omega$, où $\omega$ désigne la forme de Fubini-Study. La mesure de Green, notée $\mu$, est une mesure de probabilité invariante, obtenue comme $k$-ième puissance extérieure de $T$. Ces objets, introduits par Hubbard-Papadopol [15] et Fornaess-Sibony [12], possèdent de remarquables propriétés ergodiques. Fornaess et Sibony ont montré que la mesure de Green est mélangeante [13]. Briend et Duval ont établi que ses exposants de Liapounov sont supérieurs à $\log \sqrt{d}$ [5] et qu'elle est l'unique mesure d'entropie maximale de $f$ [6].

La dimension de $\mu$, notée $\operatorname{dim}(\mu)$, est définie comme la borne inférieure des dimensions de Hausdorff des boréliens de $\mu$-mesure pleine. C'est une caractéristique géométrique importante du système dynamique $\left(\mathbb{P}^{k}, f, \mu\right)$. L'une des premières questions concernant l'estimation de cette dimension est de déterminer les systèmes pour lesquels elle est maximale ou, ce qui s'avère équivalent, ceux dont la mesure de Green 
est absolument continue par rapport à la mesure de Lebesgue $\omega^{k}$. L'objet de cet article est de caractériser ces systèmes, ce qui répond à une question posée par Fornaess et Sibony dans [14] :

Théorème 1. Les seuls endomorphismes holomorphes de $\mathbb{P}^{k}$ dont la mesure de Green est absolument continue par rapport à $\omega^{k}$ sont les endomorphismes de Lattès.

Rappelons qu'un endomorphisme $f$ est de Lattès si il fait commuter un diagramme :

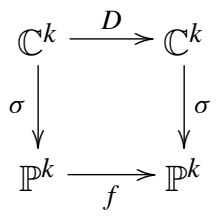

où $D$ est une application affine de partie linéaire $\sqrt{d} U$ (où $U$ est unitaire) et $\sigma$ un revêtement ramifié sur les fibres duquel un groupe cristallographique complexe agit transitivement. De tels endomorphismes existent en toute dimension $k$ et tout degré $d$; leur mesure de Green est absolument continue par rapport à $\omega^{k}$ d'exposants de Liapounov égaux à $\log \sqrt{d}$ [10]. En dimension 1, ils coïncident avec les fractions rationnelles induites par une isogénie d'un tore complexe au moyen d'une fonction elliptique. Ils sont traditionnellement appelés «exemples de Lattès» et font l'objet d'une étude détaillée dans l'article de revue de Milnor [22]. Signalons enfin que les endomorphismes de Lattès interviennent naturellement dans d'autres problèmes, comme celui de la densité des fractions rationnelles hyperboliques via la «conjecture NILF» (voir [19], [3] Chap. 7) ou celui de la classification des paires d'endomorphismes qui commutent [8]. Ils fournissent également des exemples surprenants de domaines de $\mathbb{C}^{k+1}$ munis d'auto-applications holomorphes propres non injectives [10].

Voyons comment le théorème 1 se traduit en terme de dimension de la mesure. La maximalité de la dimension entraîne la minimalité des exposants. Cela résulte de l'inégalité $\operatorname{dim}(\mu) \leq 2(k-1)+\frac{\log d}{\lambda_{k}}$, où $\lambda_{k}$ désigne le plus grand exposant de $\mu$. Cette estimation, dont la preuve est esquissée en appendice, est due à Binder et DeMarco [4] pour les applications polynomiales (voir aussi [7] pour un résultat plus précis). Il est alors possible d'adapter aux dimensions supérieures le travail de Ledrappier [17], [18] selon lequel, pour toute fraction rationnelle, l'égalité dans la formule de MargulisRuelle entraîne l'absolue continuité de $\mu$. Cela fait l'objet de [11] et concerne en particulier les mesures de Green d'exposants minimaux. Le théorème 1 admet donc pour corollaire :

Corollaire 1. Soit un système $\left(\mathbb{P}^{k}, f, \mu\right)$ où $f$ est de degréd. Les propriétés suivantes sont équivalentes :

1. La dimension de $\mu$ est maximale, égale à $2 k$. 
2. Les exposants de $\mu$ sont minimaux, égaux à $\log \sqrt{d}$.

3. L'endomorphisme $f$ est de Lattès.

Ainsi, pour un système $\left(\mathbb{P}^{k}, f, \mu\right)$ générique, la mesure $\mu$ est singulière par rapport à $\omega^{k}$, l'un de ses exposants est strictement supérieur à $\log \sqrt{d}$ et sa dimension est strictement inférieure à $2 k$.

En dimension $k=1$, on trouve une démonstration du théorème 1 dans l'article de Mayer [21]. Signalons aussi le résultat beaucoup plus précis de Zdunik [24] qui stipule que la dimension de $\mu$ coïncide avec celle de son support (l'ensemble de Julia de $f$ ) si et seulement si $f$ est un exemple de Lattès, un polynôme de Tchebychev ou une puissance $z^{ \pm d}$. La démonstration de Mayer repose sur un procédé de linéarisation consistant à comparer les itérées $f^{n}$ avec leurs applications linéaires tangentes $d_{x} f^{n}$. Un tel procédé permet de «régulariser» la densité mesurable de $\mu$ : celle-ci est en fait lisse sur un ouvert. La structure de $f$ se lit alors sur l'équation fonctionnelle $f^{*} \mu=d \mu$.

Il y a plusieurs difficultés à surmonter en dimension supérieure. Fondamentalement, le problème tient à ce que la mesure $\mu$ ne porte pas les informations géométriques «directionnelles» nécessaires à l'analyse de la structure de $f$ : celles-ci sont recelées par le courant $T$ dont elle dérive $\left(\mu=T^{k}\right)$ et s'y lisent particulièrement bien lorsque celui-ci est lisse :

Théorème (Berteloot-Loeb [2]). Tout endomorphisme holomorphe de $\mathbb{P}^{k}$ dont le courant de Green coïncide avec une $(1,1)$-forme lisse strictement positive sur un ouvert est un exemple de Lattès.

Il s'agit donc de déduire la régularité du courant $T$ de l'absolue continuité de la mesure $\mu=T^{k}$. On utilise à cet effet une méthode de linéarisation locale de l'endomorphisme par des homothéties.

Techniquement, la difficulté réside dans la mise au point de cette méthode de linéarisation car il faut pallier à l'absence du théorème de Koebe.

Nous présentons maintenant la structure de l'article et les différentes étapes de la démonstration. Les résultats des sections 3 et 4 concernent la linéarisation et présentent un intérêt pour eux-mêmes. La section 3 est consacrée à la construction d'un procédé de linéarisation général. Il s'agit, pour des choix $\mu$-génériques de $x$, de rendre la suite $\left(f^{n}\right)_{n}$ normale en $x$ en la précomposant par des contractions équivalentes aux applications linéaires tangentes inverses $\left(d_{x} f^{n}\right)^{-1}$. A cet effet, nous estimons précisément les erreurs cumulées lorsque l'on remplace $f$ par sa différentielle le long d'une orbite. Outre la stricte positivité des exposants $\lambda_{1} \leq \cdots \leq \lambda_{k}$, ceci requiert l'hypothèse $\lambda_{k}<2 \lambda_{1}$. Nous obtenons le théorème suivant : 
Théorème 2. Si les exposants du système $\left(\mathbb{P}^{k}, f, \mu\right)$ sont tels que $\lambda_{k}<2 \lambda_{1}$ alors, pour $\mu$-presque tout point $x$, la suite $\left(f^{n} \circ\left(d_{x} f^{n}\right)^{-1}\right)_{n}$ possède au moins une limite injective sur un voisinage de $x$.

En vue d'obtenir un énoncé de linéarisation par des homothéties, nous majorons la norme des différentielles $\left(d_{x} f^{n}\right)^{-1}$. Ceci fait l'objet de la section 4 . Pour cela, nous reprenons la méthode pluripotentialiste de Briend et Duval [5] dans le contexte des linéarisations. Plus précisément, nous minorons la masse de l'ensemble des points $x$ où les normes $\left\|\left(d_{x} f^{n}\right)^{-1}\right\|$ sont «grandes» (voir Proposition 2). L'énoncé précis de linéarisation suivant résume les informations acquises dans cette section sous une forme maniable.

Théorème 3. Si les exposants du système $\left(\mathbb{P}^{k}, f, \mu\right)$ sont tels que $\lambda_{k}<2 \lambda_{1}$, alors pour tout borélien $B$, il existe un borélien $\tilde{B} \subset B$ de masse arbitrairement proche de $\mu(B)^{2}$ et $\tau_{0}>0$ vérifiant les assertions suivantes : pour tout point $x \in \tilde{B}$, il existe une suite extraite $\left(f^{n_{j}}\right)_{j}$ et un réel $v(x)>0$ tels que

1. $f^{n_{j}}(x) \in$ B pour tout $j \in \mathbb{N}$.

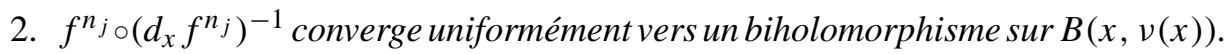

3. $\left\|\left(d_{x} f^{n_{j}}\right)^{-1}\right\| \leq \tau_{0}(\sqrt{d})^{-n_{j}}$ pour tout $j \in \mathbb{N}$.

Dans la section 5, nous montrons que si la mesure $\mu$ est absolument continue alors les différentielles $\left(d_{x} f^{n_{j}}\right)^{-1}$ intervenant dans le théorème 3 sont équivalentes à des homothéties de rapport $(\sqrt{d})^{-n_{j}}$. Notons que la condition $\lambda_{k}<2 \lambda_{1}$ est satisfaite car la régularité de $\mu$ entraîne la minimalité des exposants.

Nous achevons la preuve du théorème 1 dans la section 6 . Nous montrons que le courant $T$ est régulier en utilisant le procédé de linéarisation par les homothéties de rapport $(\sqrt{d})^{-n}$ et les relations d'invariance $f^{n *} T=d^{n} T$. Le résultat de [2] cité plus haut montre qu'alors $f$ est un endomorphisme de Lattès.

Remerciements. Nous tenons à remercier le rapporteur tant pour sa lecture attentive du manuscrit que pour ses conseils de rédaction.

\section{Préliminaires}

Nous résumons ici les principaux outils et résultats utilisés par la suite. Nous fixons également quelques notations.

2.1. Vocabulaire et notations. - Un système $\left(\mathbb{P}^{k}, f, \mu\right)$ est la donnée d'un endomorphisme holomorphe $f$ de l'espace projectif de dimension $k$ dont le degré $d$ est supérieur ou égal à 2 et dont l'unique mesure d'entropie maximale est notée $\mu$. Nous dirons aussi que le système $\left(\mathbb{P}^{k}, f, \mu\right)$ est de degré $d$. 
- Soit $\left(\widehat{\mathbb{P}^{k}}, \hat{f}, \hat{\mu}\right)$ l'extension naturelle du système $\left(\mathbb{P}^{k}, f, \mu\right)$. On rappelle que $\widehat{\mathbb{P}^{k}}$ est l'ensemble des orbites $\left\{\hat{x}:=\left(x_{n}\right)_{n \in \mathbb{Z}} \mid f\left(x_{n}\right)=x_{n+1}\right\}$ muni de la topologie et de la tribu produit. Soient $\pi_{0}: \widehat{\mathbb{P}}^{k} \rightarrow \mathbb{P}^{k}$ la projection définie par $\pi_{0}(\hat{x})=x_{0}, \hat{f}$ le décalage à droite et $\hat{f}^{-1}$ le décalage à gauche sur $\widehat{\mathbb{P}^{k}}$, de sorte que $\pi_{0} \circ \hat{f}=f \circ \pi_{0}$. On note $\hat{\mu}$ l'unique mesure de probabilité $\hat{f}$-invariante sur $\widehat{P P}^{k}$ vérifiant $\pi_{0 *} \hat{\mu}=\mu$. Le caractère mélangeant de $\mu$ passe à $\hat{\mu}$.

- Soit $\widehat{X}$ le sous-ensemble de $\widehat{\mathbb{P}^{k}}$ suivant :

$$
\widehat{X}:=\left\{\hat{x} \in \widehat{\mathbb{P}^{k}} \mid x_{n} \notin \operatorname{Crit}(f) \text { pour tout } n \in \mathbb{Z}\right\}
$$

où Crit $(f)$ désigne l'ensemble critique de $f$. Le borélien $\widehat{X}$ vérifie $\hat{\mu}(\widehat{X})=1$, car $\mu$ ne charge pas l'ensemble analytique $\operatorname{Crit}(f)$ ([23], Proposition A.6.3).

Par la suite, on s'autorisera à soustraire à $\widehat{X}$ des ensembles $\hat{\mu}$-négligeables.

2.2. Branches inverses et exposants. - On construit une famille de cartes holomorphes $\left(\tau_{x}\right)_{x \in \mathbb{P}^{k}}$ telle que :

1. $\tau_{x}: \mathbb{C}^{k} \rightarrow \mathbb{P}^{k}$ est un biholomorphisme sur son image et $\tau_{x}(0)=x$,

2. $\left(\tau_{x}^{*} \omega\right)_{0}=\frac{i}{2} \sum_{j=1, k} d z_{j} \wedge d \bar{z}_{j}$.

où $\omega$ désigne la forme de Fubini-Study. Cette famille est obtenue en explicitant une telle carte en un point base $x_{0} \in \mathbb{P}^{k}$, puis en la propageant à $\mathbb{P}^{k}$ par l' action transitive de $\mathrm{U}_{k+1}(\mathbb{C})$. Ce faisant, on obtient plutôt une classe de cartes en $x$ car $\tau_{x}$ est définie à un élément du sous-groupe d'isotropie de $x_{0}$ près. Cette ambiguïté pourra cependant être ignorée puisque $\mathrm{U}_{k+1}(\mathbb{C})$ est compact ; les affirmations faisant intervenir $\tau_{x}$ devront être comprises comme valables pour tous les éléments de la classe de cartes en $x$. On peut aussi, localement, faire un choix «différentiable» de $\tau_{x}$ par rapport à $x$ et en particulier s'assurer que la propriété suivante est vérifiée :

$(\star) \tau_{x_{0}}^{-1} \circ \tau_{x}-\tau_{x_{0}}^{-1}(x)$ converge vers l'identité en topologie $\mathrm{C}^{\infty}$ lorsque $x$ tend vers $x_{0}$.

- Nous noterons $B(0, R)$ (resp. $P(0, R))$ la boule euclidienne (resp. le polydisque) de $\mathbb{C}^{k}$ centrée en 0 et de rayon $R$ (resp. de polyrayon $(R, \ldots, R)$ ). On désignera par $B(x, s)$ l'image de $B(0, s)$ par $\tau_{x}$.

- Nous utiliserons les applications suivantes, où $x \in \mathbb{P}^{k}$ et $n \in \mathbb{N}$ :

$$
\begin{gathered}
f_{x}:=\tau_{f(x)}^{-1} \circ f \circ \tau_{x} \\
f_{x}^{n}=\tau_{f^{n}(x)}^{-1} \circ f^{n} \circ \tau_{x}=f_{f^{n}(x)} \circ \cdots \circ f_{x} .
\end{gathered}
$$

Elles sont définies sur un voisinage de l'origine de $\mathbb{C}^{k}$, dont la taille dépend de $x$ et de $n$. Pour tout $\hat{x} \in \widehat{X}$, on note $f_{\hat{x}}^{-n}$ la branche inverse de $f^{n}$ «le long de l'orbite $\hat{x}$, c'est-à-dire :

$$
f_{\hat{x}}^{-n}:=f_{x_{-n}}^{-1} \circ \cdots \circ f_{x_{-1}}^{-1}
$$


Le lemme suivant stipule que ces branches inverses existent sur un voisinage de l'origine dont la taille dépend mesurablement de $\hat{x}$. On trouvera une preuve dans l'article de Briend-Duval [5] (voir aussi [9] pp. 19-22).

Lemme 1. Soient $0<\epsilon \ll 1$ et $0<r_{0} \ll 1$. Il existe des fonctions $\rho$ et $r$ continues sur $\mathbb{P}^{k}$ strictement positives hors de $\operatorname{Crit}(f)$, ainsi que des fonctions mesurables $\left.\eta: \widehat{X} \rightarrow] 0, r_{0}\right]$ et $C: \widehat{X} \rightarrow[1,+\infty[$ vérifiant les propriétés suivantes :

1. Pour tout $x \in \mathbb{P}^{k} \backslash \operatorname{Crit}(f), f_{x}$ est injective sur $B(0, \rho(x))$ et

$$
B(0, r(x)) \subset f_{x}[B(0, \rho(x))] .
$$

2. Pour tout $\hat{x} \in \widehat{X}, \lim _{n} \frac{1}{n} \log \rho\left(x_{n}\right)=0$.

3. Pour tout $\hat{x} \in \widehat{X}$ et tout $n \in \mathbb{N}, f_{\hat{x}}^{-n}$ est injective sur $B(0, \eta(\hat{x}))$, et pour tout $\gamma \in] 0,1]$

$$
d_{0} f_{\hat{x}}^{-n}[B(0, \gamma \eta(\hat{x}))] \subset B\left(0, \gamma r\left(x_{-(n+1)}\right) e^{-n\left(\lambda_{1}-\epsilon\right)}\right) .
$$

4. $\operatorname{Lip} f_{\hat{x}}^{-n} \leq C(\hat{x}) e^{-n\left(\lambda_{1}-\frac{\epsilon}{2}\right)} \operatorname{sur} B(0, \eta(\hat{x}))$.

- Les exposants de Liapounov de $\mu$ seront notés $\lambda_{1} \leq \cdots \leq \lambda_{k}$. Nous utiliserons de manière cruciale la minoration optimale de ces exposants :

Théorème (Briend-Duval [5]). Les exposants d'un système $\left(\mathbb{P}^{k}, f, \mu\right)$ de degré $d$ sont plus grands que $\log \sqrt{d}$.

\section{Un procédé de linéarisation}

Notre objectif est de démontrer le théorème 2 présenté dans l'introduction. Nous adoptons la définition suivante :

Définition 1. Un système $\left(\mathbb{P}^{k}, f, \mu\right)$ est dit linéarisable si pour $\mu$-presque tout $x \in$ $\mathbb{P}^{k}$, il existe $v(x)>0$ et une sous-suite de $\left[f^{n} \circ \tau_{x} \circ\left(d_{0} f_{x}^{n}\right)^{-1}\right]_{n}$ qui converge uniformément vers une limite injective sur $B(0, v(x))$.

La proposition suivante fournit deux conditions suffisantes de linéarisibilité. La première réduit le problème au contrôle uniforme local de la suite $f_{x}^{n} \circ\left(d_{0} f_{x}^{n}\right)^{-1}$ grâce au théorème de Montel. La seconde transfère cette question de contrôle uniforme en «temps négatif», c'est à dire aux applications $f_{x_{-n}}^{n} \circ d_{0} f_{\hat{x}}^{-n}$. Nous utilisons pour cela un argument classique basé sur l'invariance de la mesure $\hat{\mu}$. 
Proposition 1. Soit $\left(\mathbb{P}^{k}, f, \mu\right)$ un système et $R_{0}$ un nombre réel strictement positif. Pour tout $\rho \in] 0,1]$ et $n \in \mathbb{N}$, on définit les ensembles :

$$
\begin{gathered}
\mathcal{B}_{n}(\rho):=\left\{x \in \mathbb{P}^{k} \mid f_{x}^{n} \circ\left(d_{0} f_{x}^{n}\right)^{-1} \text { est injective de } B(0, \rho) \text { dans } B\left(0, R_{0}\right)\right\} \\
\mathcal{B}(\rho):=\limsup _{n} \mathcal{B}_{n}(\rho) .
\end{gathered}
$$

Le système est linéarisable si l'une des deux conditions suivantes est réalisée :

1) Il existe $\alpha:] 0,1] \rightarrow \mathbb{R}^{+}$telle que $\lim _{\rho \rightarrow 0} \alpha(\rho)=1$ et $\mu\left[\mathscr{B}_{n}(\rho)\right] \geq \alpha(\rho)$ pour tout $n \in \mathbb{N}$.

2) Pour tout $\left.\left.r_{0} \in\right] 0, R_{0}\right]$ il existe des fonctions mesurables $\left.\left.\eta, S: \widehat{X} \rightarrow\right] 0, r_{0}\right]$ telles que

(i) $S \leq \eta$.

(ii) Pour tout $\hat{x} \in \widehat{X}, f_{\hat{x}}^{-n}$ est injective sur $B(0, \eta(\hat{x}))$.

(iii) Pour tout $\hat{x} \in \widehat{X}$ et tout $n \in \mathbb{N}, d_{0} f_{\hat{x}}^{-n}[B(0, S(\hat{x}))] \subset f_{\hat{x}}^{-n}[B(0, \eta(\hat{x}))]$.

La seconde assertion implique la première.

Démonstration. La linéarisabilité en $x$ résulte, via le théorème de Montel, de l'appartenance de $x$ à $\bigcup_{0<\rho \leq 1} \mathscr{B}(\rho)$. Ainsi, comme $\mu[\mathscr{B}(\rho)] \geq \lim \sup _{n} \mu\left[\mathscr{B}_{n}(\rho)\right]$, la condition 1 entraîne la linéarisabilité $\mu$-presque partout.

Voyons maintenant comment la seconde condition entraîne la première. Posons $\widehat{\jmath}(\rho):=\{\hat{x} \in \widehat{X} \mid S(\hat{x}) \geq \rho\}$. Il suffit d'établir les inclusions suivantes :

$$
\pi_{0}\left[\hat{f}^{-n}(\widehat{\delta}(\rho))\right] \subset \mathcal{B}_{n}(\rho) \quad \text { pour tout } n \in \mathbb{N} .
$$

En effet, compte tenu de l'invariance de $\hat{\mu}$, on a $\mu\left[\mathscr{B}_{n}(\rho)\right] \geq \hat{\mu}\left[\hat{f}^{-n}(\widehat{\delta}(\rho))\right]=$ $\hat{\mu}[\widehat{\jmath}(\rho)]$. La fonction $\alpha(\rho):=\hat{\mu}[\widehat{\jmath}(\rho)]$ convient car $S$ est strictement positive $\hat{\mu}$ presque partout.

Établissons maintenant les inclusions annoncées. Soit $\hat{y}:=\hat{f}^{n}(\hat{x})$ tel que $\hat{y} \in$ $\widehat{\delta}(\rho)$. Il s'agit de vérifier que $x_{0} \in \mathscr{B}_{n}(\rho)$. Rappelons que $x_{0}=\pi_{0}(\hat{x})$. L'appartenance de $\hat{y}$ à $\widehat{\delta}(\rho)$ signifie :

$$
d_{0} f_{\hat{y}}^{-n}[B(0, \rho)] \subset d_{0} f_{\hat{y}}^{-n}[B(0, S(\hat{y}))] \subset f_{\hat{y}}^{-n}[B(0, \eta(\hat{y})] .
$$

Comme $f_{\hat{y}}^{-n}$ est injective sur $B(0, \eta(\hat{y}))$ d'inverse $f_{x_{0}}^{n}$, on obtient en composant les inclusions précédentes par $f_{x_{0}}^{n}$ :

$$
f_{x_{0}}^{n} \circ\left(d_{0} f_{x_{0}}^{n}\right)^{-1}[B(0, \rho)] \subset B(0, \eta(\hat{y})) \subset B\left(0, R_{0}\right) .
$$

Le point $x_{0}$ appartient donc à $\mathscr{B}_{n}(\rho)$. 
Nous démontrerons le théorème 2 en vérifiant que la condition 2 de la proposition 1 est satisfaite. Ceci consistera à compenser les erreurs dues à la substitution de $d_{0} f_{x_{j}}^{-1}$ à $f_{x_{j}}^{-1}$ le long de $\hat{x}$ en diminuant le rayon $\eta(\hat{x})$. Pour que les compensations cumulées fournissent un rayon $S(\hat{x})$ strictement positif, les erreurs commises devront être négligeables devant la plus petite dimension caractéristique de l'ellipsoïde $d_{0} f_{\hat{x}}^{-j}[B(0,1)]$. L'objet du lemme suivant est de montrer que tel est le cas lorsque les exposants vérifient l'inégalité $\lambda_{k}<2 \lambda_{1}$.

Lemme 2. Soient un système $\left(\mathbb{P}^{k}, f, \mu\right)$ et $0<\epsilon \ll 1$. Il existe des fonctions mesurables $\eta, E, F: \widehat{X} \rightarrow] 0,+\infty\left[\right.$ vérifiant $0<\eta \leq r_{0} \leq R_{0}$ telles que pour tout $\hat{x}=\left(x_{n}\right)_{n \in \mathbb{Z}}$ élément de $\widehat{X}$ et tout $n \in \mathbb{N}$ :

1. $f_{\hat{x}}^{-n}$ est injective sur $B(0, \eta(\hat{x}))$.

2. Pour tout $\gamma \in] 0,1]$ et tout $u \in d_{0} f_{\hat{x}}^{-n}[B(0, \gamma \eta(\hat{x}))]$ :

$$
\left\|\left(d_{0} f_{x_{-(n+1)}}^{-1}-f_{x_{-(n+1)}}^{-1}\right)(u)\right\| \leq \gamma E(\hat{x}) e^{-2 n\left(\lambda_{1}-\epsilon\right)} .
$$

3. $\left\|d_{0} f_{x_{-(n+1)}}^{n+1}\right\| \leq F(\hat{x}) e^{n\left(\lambda_{k}+\epsilon\right)}$.

Démonstration. Nous utilisons ici le lemme 1. Pour tout $\hat{x} \in \widehat{X}$, l'assertion 1 est satisfaite. De plus, l'application $f_{x_{-(n+1)}}$ est inversible sur $B(0, r)$, où $r:=r\left(x_{-(n+1)}\right)$, et son inverse $g$ est à valeurs dans $B(0, \rho)$ où $\rho:=\rho\left(x_{-(n+1)}\right)$. Soit $\sum_{p \geq 2} Q_{p}$ le développement de Taylor de $g-d_{0} g$, où $Q_{p}$ désigne une application homogène de degré $p$. Si $u \in B(0, r)$ alors $\left\|Q_{p}(u)\right\|=\left\|\frac{1}{2 \pi} \int_{0}^{2 \pi} g\left(e^{i \theta} u\right) e^{-i p \theta} d \theta\right\| \leq \rho$ et donc :

$$
\left\|\left(g-d_{0} g\right)(u)\right\| \leq \sum_{p \geq 2} \frac{\|u\|^{p}}{r^{p}}\left\|Q_{p}\left(\frac{r u}{\|u\|}\right)\right\| \leq \rho \sum_{p \geq 2}\left(\frac{\|u\|}{r}\right)^{p} .
$$

Lorsque de plus $u \in d_{0} f_{\hat{x}}^{-n}\left[B(0, \gamma \eta(\hat{x})]\right.$ alors $\frac{\|u\|}{r} \leq \gamma e^{-n\left(\lambda_{1}-\epsilon\right)}$ (cf lemme 1, (3)) et il s'ensuit que :

$$
\left\|\left(g-d_{0} g\right)(u)\right\| \leq \frac{\gamma^{2} \rho}{1-\gamma e^{-\left(\lambda_{1}-\epsilon\right)}} e^{-2 n\left(\lambda_{1}-\epsilon\right)} .
$$

L'assertion 2 du lemme s'en déduit car $\rho\left(x_{-(n+1)}\right)$ a un taux de croissance exponentiel nul (cf lemme 1,(2)). La dernière assertion découle immédiatement de la définition des exposants de Liapounov. Nous ôtons ici à $\widehat{X}$ un sous-ensemble de $\hat{\mu}$ mesure nulle.

Démonstration du théorème 2. Il s'agit de montrer que la condition 2 de la proposition 1 est satisfaite lorsque $\lambda_{k}<2 \lambda_{1}$. Reprenons les notations du lemme 2 et introduisons sur $\widehat{X}$ les fonctions mesurables suivantes :

$$
\xi_{n}(\hat{x}):=\sup \left\{t \leq \eta(\hat{x}) \mid d_{0} f_{\hat{x}}^{-n}[B(0, t)] \subset f_{\hat{x}}^{-n}[B(0, \eta(\hat{x}))]\right\}
$$


Vol. 80 (2005)

$$
\begin{aligned}
n_{0}(\hat{x}):= & \min \left\{p \geq 1 \mid \text { pour tout } n \geq p: \frac{E F}{\eta}(\hat{x}) \leq e^{n \epsilon}\right\} \\
& s(\hat{x}):=\min \left\{\xi_{n}(\hat{x}) \mid 0 \leq n \leq n_{0}(\hat{x})\right\} .
\end{aligned}
$$

Posons $\kappa_{j}:=1-e^{-j\left(2 \lambda_{1}-\lambda_{k}-6 \epsilon\right)}$ avec $\epsilon$ suffisamment petit pour que le produit $\prod_{j=1}^{\infty} \kappa_{j}$ converge et soit strictement positif. Définissons les fonctions $s_{n}$ par :

$$
\begin{array}{lll}
s_{n}(\hat{x}):=s(\hat{x}) & \text { si } n \leq n_{0}(\hat{x}) \\
s_{n}(\hat{x}):=s(\hat{x}) \prod_{j=n_{0}(\hat{x})}^{n-1} \kappa_{j} & \text { si } n \geq n_{0}(\hat{x})+1 .
\end{array}
$$

Pour montrer que la fonction $S(\hat{x}):=s(\hat{x}) \prod_{j=1}^{\infty} \kappa_{j}$ convient, il suffit d'établir les inclusions :

$$
\left(I_{n}\right)_{n \geq 0}: \quad d_{0} f_{\hat{x}}^{-n}\left[B\left(0, s_{n}(\hat{x})\right)\right] \subset f_{\hat{x}}^{-n}[B(0, \eta(\hat{x}))] .
$$

Par définition de $s_{n}(\hat{x})$, ces inclusions sont satisfaites lorsque $n \leq n_{0}(\hat{x})$. Supposons que $\left(I_{n}\right)$ soit vraie pour $n \geq n_{0}(\hat{x})$ et posons $v_{n}:=\left(\frac{E s_{n}}{\eta}\right)(\hat{x}) e^{-2 n\left(\lambda_{1}-2 \epsilon\right)}$. On a alors :

$$
s_{n+1} \leq s_{n}-\left\|\left(d_{0} f_{\hat{x}}^{-(n+1)}\right)^{-1}\right\| v_{n} .
$$

En effet :

$$
\begin{aligned}
s_{n+1}=s_{n} \kappa_{n} & =s_{n}\left(1-e^{-n\left(2 \lambda_{1}-\lambda_{k}-6 \epsilon\right)}\right) \leq s_{n}\left(1-\frac{E F}{\eta}(\hat{x}) e^{-n\left(2 \lambda_{1}-\lambda_{k}-5 \epsilon\right)}\right) \\
& \leq s_{n}-\left\|d_{0} f_{x_{-(n+1)}^{n+1}}^{n+1}\right\| v_{n}=s_{n}-\left\|\left(d_{0} f_{\hat{x}}^{-(n+1)}\right)^{-1}\right\| v_{n}
\end{aligned}
$$

la première majoration résultant de la définition de $n_{0}(\hat{x})$ et la seconde du lemme 2, (2).

Désignons par $\Lambda$ la frontière de $d_{0} f_{\hat{x}}^{-(n+1)}\left[B\left(0, s_{n}\right)\right]$. On vérifie aisément que l'inégalité (1) se traduit par :

$$
d_{0} f_{\hat{x}}^{-(n+1)}\left[B\left(0, s_{n+1}\right)\right] \subset d_{0} f_{\hat{x}}^{-(n+1)}\left[B\left(0, s_{n}\right)\right] \backslash \bigcup_{p \in \Lambda} B\left(p, v_{n}\right) .
$$

Par ailleurs, la première assertion du lemme 2 (où l'on prend $\gamma=\frac{s_{n}}{\eta}$ ) stipule que sur $d_{0} f_{\hat{x}}^{-n}\left[B\left(0, s_{n}\right)\right], f_{x_{-(n+1)}}^{-1}$ diffère d'au plus $v_{n}$ de sa différentielle. Il s'ensuit que

$$
d_{0} f_{\hat{x}}^{-(n+1)}\left[B\left(0, s_{n}\right)\right] \backslash \bigcup_{p \in \Lambda} B\left(p, v_{n}\right) \subset f_{x_{-(n+1)}}^{-1} \circ d_{0} f_{\hat{x}}^{-n}\left[B\left(0, s_{n}\right)\right] .
$$

Observons finalement que l'inclusion $\left(I_{n}\right)$, composée par $f_{x_{-(n+1)}}^{-1}$, s'écrit

$$
f_{x_{-(n+1)}}^{-1} \circ d_{0} f_{\hat{x}}^{-n}\left[B\left(0, s_{n}\right)\right] \subset f_{\hat{x}}^{-(n+1)}[B(0, \eta(\hat{x})] .
$$

Les inclusions (2), (3) et (4) enchaînées donnent $\left(I_{n+1}\right)$. 
Remarque 1. L'inégalité $\lambda_{k}<2 \lambda_{1}$ peut être interprêtée comme une condition de non résonance entraînant la linéarisabilité. Jonsson et Varolin (cf [16], Theorem 3) ont, indépendamment de nous, mis en évidence la même condition dans un problème voisin.

\section{Une version précisée du procédé de linéarisation}

L'objet de cette section est de contrôler le diamètre des ellipsoïdes $\left(d_{0} f_{x}^{n}\right)^{-1}[B(0,1)]$ associés au procédé de linéarisation fourni par le théorème 2 . Nous en déduisons le théorème 3 énoncé dans l'introduction.

Le théorème de Briend-Duval, déjà utilisé implicitement pour établir le lemme 1, majore le taux de décroissance exponentielle de la taille de ces ellipsoïdes par $-\log \sqrt{d}$. Cela signifie que pour tout $\epsilon>0$, on a $\left\|\left(d_{0} f_{x}^{n}\right)^{-1}\right\| \lesssim e^{n \epsilon}(\sqrt{d})^{-n}$ pour $n$ assez grand. En reprenant la méthode de Briend-Duval dans le contexte de la proposition 1, nous obtenons une majoration plus précise : $\left\|\left(d_{0} f_{x}^{n}\right)^{-1}\right\| \lesssim(\sqrt{d})^{-n}$. Rappelons que $\mathscr{B}_{n}(\rho)$ est défini par :

$$
\mathcal{B}_{n}(\rho):=\left\{x \in \mathbb{P}^{k} \mid f_{x}^{n} \circ\left(d_{0} f_{x}^{n}\right)^{-1} \text { est injective de } B(0, \rho) \text { dans } B\left(0, R_{0}\right)\right\}
$$

et qu'en vertu de la proposition 1 et de la preuve du théorème 2 , il existe une fonction $\alpha:] 0,1] \rightarrow \mathbb{R}^{+}$telle que $\lim _{\rho \rightarrow 0} \alpha(\rho)=1$ et

$$
\mu\left[\mathscr{B}_{n}(\rho)\right] \geq \alpha(\rho) .
$$

Nous montrons la proposition suivante :

Proposition 2. Soit $\left(\mathbb{P}^{k}, f, \mu\right)$ un système de degré $d \geq 2$ tel que $\lambda_{k}<2 \lambda_{1}$. On pose pour $\tau>0, \rho \in] 0,1]$ et $n \in \mathbb{N}$ :

$$
\mathscr{D}_{n}(\rho, \tau):=\mathscr{B}_{n}(\rho) \cap\left\{x \in \mathbb{P}^{k} \mid\left\|\left(d_{0} f_{x}^{n}\right)^{-1}\right\| \leq \tau(\sqrt{d})^{-n}\right\} .
$$

Alors on a l'inégalité :

$$
\liminf _{n} \mu\left[\mathscr{D}_{n}(\rho, \tau)\right] \geq \alpha(\rho)-\frac{C}{\tau^{2} \rho^{2}},
$$

où $C>0$ et $\alpha:] 0,1] \rightarrow \mathbb{R}^{+}$est une fonction telle que $\lim _{\rho \rightarrow 0} \alpha(\rho)=1$.

Le principe de la preuve est le suivant. Puisque $\mu\left[\mathcal{B}_{n}(\rho)\right] \geq \alpha(\rho)$ d'après la proposition 1, il s'agit de majorer la mesure du complémentaire de $\mathscr{D}_{n}(\rho, \tau)$ dans $\mathscr{B}_{n}(\rho)$, noté $\mathscr{D}_{n}^{c}(\rho, \tau)$. Or, par tout point de $\mathscr{D}_{n}^{c}(\rho, \tau)$ passe un disque dont le diamètre est au moins égal à $\tau \rho(\sqrt{d})^{-n}$ et dont l'image par $f^{n}$ reste contenue dans une boule de rayon $R_{0}$ fixé. Comme $f^{n *} T=d^{n} T$, il passe donc par tout point de $\mathscr{D}_{n}^{c}(\rho, \tau)$ 
un «grand» disque «peu» chargé par $T$. Des techniques pluripotentialistes permettent alors de majorer précisément la masse de l'ensemble de ces points pour la mesure $\mu=T^{k}$.

Démonstration de la proposition 2. On dira qu'un disque holomorphe $\sigma: \Delta \rightarrow \mathbb{C}^{k}$ est de taille $l>0$ et passe par $z \in \mathbb{C}^{k}$ si il est de la forme $\sigma(u)=z+l u . v+\beta(u)$ où $v$ est un vecteur unitaire de $\mathbb{C}^{k}, \beta(0)=0$ et $\|\beta\| \leq \frac{l}{1000}$.

L'ingrédient principal est le théorème suivant dont la preuve est résumée dans l'appendice :

Théorème (Briend-Duval [5]). Soit $S:=d d^{c} w$ un $(1,1)$-courant positif fermé de potentiel $w$ continu sur le polydisque $P(0, R)$ et $E \subset P\left(0, \frac{R}{2}\right)$. On suppose que par tout point $z \in E$ passe un disque holomorphe $\sigma_{z}: \Delta \rightarrow \mathbb{C}^{k}$ de taille l et qu'il existe une fonction $h_{z}$ harmonique sur $\Delta$ telle que $\left|w \circ \sigma_{z}-h_{z}\right| \leq \epsilon$ sur $\Delta$. Alors il existe une constante $C(w)$ ne dépendant que de $w$ telle que $S^{k}(E) \leq C(w) \frac{k^{2}}{l^{2}} \epsilon$.

En vue d'utiliser ce résultat, nous fixons des systèmes de coordonnées locales sur $\mathbb{P}^{k}$. Considérons un recouvrement de $\mathbb{P}^{k}$ par des ouverts $U_{1}, \ldots, U_{N}$ centrés en des points $m_{j}$ et tel que sur chaque $U_{j}$ nous puissions fixer des déterminations des cartes $\tau_{x}$ dépendant différentiablement de $x$ (cf la condition ( $\star$ ), section 2). Posons $\tau_{j}:=\tau_{m_{j}}$ puis, pour $R>0$ fixé, $V_{j}:=\tau_{j}(P(0, R))$. Si le recouvrement est assez fin alors les propriétés suivantes sont satisfaites :

(i) $U_{j} \subset \tau_{j}\left(P\left(0, \frac{R}{2}\right)\right)$ et $\tau_{x}\left(P\left(0, \frac{R}{2}\right)\right) \subset V_{j}$ pour tout $x \in U_{j}$,

(ii) $\left\|\tau_{j}^{-1} \circ \tau_{x}-\left(\tau_{j}^{-1}(x)+\operatorname{Id}_{\mathbb{C}^{\mathrm{k}}}\right)\right\|_{\mathcal{C}^{1}, \overline{P\left(0, \frac{R}{2}\right)}} \leq \frac{1}{1000}$ pour tout $x \in U_{j}$.

Puis, si $R_{0}$ (introduit au lemme 1) est pris assez petit :

(iii) pour tout $x \in \mathbb{P}^{k}$ il existe $l \in\{1, \ldots, N\}$ tel que $\tau_{x}\left[B\left(0, R_{0}\right)\right] \subset V_{l}$,

(iv) $\mu\left\{x \in U_{j} \cap \mathscr{B}_{n}(\rho) \mid\left(d_{0} f_{x}^{n}\right)^{-1}[B(0, \rho)] \subset P\left(0, \frac{R}{2}\right)\right\}=\mu\left(U_{j} \cap \mathscr{B}_{n}(\rho)\right)-\epsilon_{n, j}$ avec $\lim _{n} \epsilon_{n, j}=0$.

Enfin, si $v_{j}$ désigne un potentiel continu de $T$ sur $V_{j}$, il existe une constante $M>0$ telle que :

(v) $T=d d^{c} v_{j}$ et $\left|v_{j}\right| \leq M$ sur $V_{j}$ pour tout $j \in\{1, \ldots, N\}$.

D'après le théorème 2 , il existe une fonction $\alpha$ qui vérifie la propriété énoncée à la proposition $1(1)$; autrement dit, $\alpha(\rho)$ tend vers 1 quand $\rho$ tend vers 0 et $\mu\left[\mathscr{B}_{n}(\rho)\right] \geq$ $\alpha(\rho)$.

Comme il s'agit de minorer $\liminf _{n} \mu\left[\mathscr{D}_{n}(\rho, \tau)\right]$, la propriété (iv) montre que l'on peut considérer que :

$$
\left(d_{0} f_{x}^{n}\right)^{-1}[B(0, \rho)] \subset P\left(0, \frac{R}{2}\right) \text { pour tout } x \in U_{j} \cap \mathcal{B}_{n}(\rho) .
$$


Pour tout $j \in\{1, \ldots, N\}$ nous allons établir que :

$$
\mu\left[\mathscr{D}_{n}^{c}(\rho, \tau) \cap U_{j}\right] \leq C\left(v_{j} \circ \tau_{j}\right) \frac{M k^{2}}{\tau^{2} \rho^{2}} .
$$

Soit donc $x \in \mathscr{D}_{n}^{c}(\rho, \tau) \cap U_{j}$ et $V_{n}(x)$ un vecteur unitaire tel que $\left\|\left(d_{0} f_{x}^{n}\right)^{-1}\right\|=$ $\left\|\left(d_{0} f_{x}^{n}\right)^{-1}\left[V_{n}(x)\right]\right\|$. Si on note $v_{n}(x)=\left(d_{0} f_{x}^{n}\right)^{-1}\left[V_{n}(x)\right]$, on a $\left\|v_{n}(x)\right\| \geq \tau \rho(\sqrt{d})^{-n}$. On définit ainsi un disque affine $\Phi_{n, x}: \bar{\Delta} \rightarrow \mathbb{C}^{k}$ de diamètre au moins égal à $\tau \rho(\sqrt{d})^{-n}$ en posant :

$$
\Phi_{n, x}(t):=\left(d_{0} f_{x}^{n}\right)^{-1}\left[t \rho . V_{n}(x)\right]=t \rho . v_{n}(x) .
$$

Comme $x \in U_{j}$, (5) et (i) permettent de définir un nouveau disque $\Phi_{j, n, x}: \Delta \rightarrow$ $P(0, R) \operatorname{par} \Phi_{j, n, x}:=\tau_{j}^{-1} \circ \tau_{x} \circ \Phi_{n, x}$. Compte tenu de la propriété (ii), $\Phi_{j, n, x}$ est un disque holomorphe de taille $l:=\rho\left\|v_{n}(x)\right\| \geq \tau \rho(\sqrt{d})^{-n}$ passant par $\tau_{j}^{-1}(x)$.

Choisissons $l \in\{1, \ldots, N\}$ tel que $\tau_{f^{n}(x)}\left[B\left(0, R_{0}\right)\right] \subset V_{l}$ (propriété (iii)) alors, comme $x \in \mathscr{B}_{n}(\rho)$, on a $f^{n} \circ \tau_{x} \circ \Phi_{n, x}(\Delta) \subset \tau_{f^{n}(x)}\left[B\left(0, R_{0}\right)\right] \subset V_{l}$ et donc

$d d^{c}\left(v_{l} \circ f^{n} \circ \tau_{x} \circ \Phi_{n, x}\right)=\left(f^{n} \circ \tau_{x} \circ \Phi_{n, x}\right)^{*} T=\left(\tau_{x} \circ \Phi_{n, x}\right)^{*} f^{n *} T=d^{n}\left(\tau_{x} \circ \Phi_{n, x}\right)^{*} T$.

Par ailleurs, puisque $\tau_{x} \circ \Phi_{n, x}(\Delta) \subset V_{j}$ (cf. (5) et (i)), on a

$$
\left(\tau_{x} \circ \Phi_{n, x}\right)^{*} T=d d^{c}\left(v_{j} \circ \tau_{x} \circ \Phi_{n, x}\right)=d d^{c}\left(v_{j} \circ \tau_{j} \circ \Phi_{j, n, x}\right) .
$$

Ainsi, $d d^{c}\left[v_{l} \circ f^{n} \circ \tau_{x} \circ \Phi_{n, x}-d^{n} v_{j} \circ \tau_{j} \circ \Phi_{j, n, x}\right]=0$. Autrement dit, la fonction entre crochets est harmonique sur $\Delta$ et, puisque $\left|v_{l}\right| \leq M$, le potentiel $v_{j} \circ \tau_{j}$ de $\tau_{j}^{*} T$ diffère d'au plus $\frac{M}{d^{n}}$ d'une fonction harmonique $h$ sur le disque $\Phi_{j, n, x}$ de taille $l \geq \tau \rho(\sqrt{d})^{-n}$. Dans ces conditions, (6) découle immédiatement du théorème de Briend-Duval. On en déduit l'estimation annoncée avec $C=M k^{2} \sum_{j=1}^{N} C\left(v_{j} \circ \tau_{j}\right)$.

Terminons cette section par la preuve du théorème 3. Il s'agit d'établir une version du procédé de linéarisation où les orbites issues d'un borélien prescrit sont assujetties à récurrence. Cette précision découle des estimations fournies par la proposition 2 et du caractère mélangeant de $\mu$.

Démonstration du théorème 3. Posons $\mathscr{D}_{n}(\rho, \tau, B):=\mathscr{D}_{n}(\rho, \tau) \cap B \cap f^{-n}(B)$ et $\mathscr{D}(\rho, \tau, B):=\lim \sup _{n} \mathscr{D}_{n}(\rho, \tau, B)$. Il est clair que si $x \in \mathscr{D}\left(\rho_{0}, \tau_{0}, B\right)$ alors il existe une suite extraite $\left(f^{n_{j}}\right)_{j}$ vérifiant les trois assertions du théorème 3 . Il suffit donc d'observer que $\mu\left(\mathscr{D}_{n}(\rho, \tau, B)\right)$ approche $\mu(B)^{2}$ par défaut pourvu que $\rho_{0}, \frac{1}{\tau_{0}}$ soient assez petits et $n$ assez grand. Or ceci résulte immédiatement de la proposition 2 et du caractère mélangeant de $\mu$. Il suffit en effet de fixer $\rho_{0}$ assez petit puis $\tau_{0}$ assez grand pour que $\mathscr{D}_{n}(\rho, \tau)$ soit presque de $\mu$-mesure pleine pour $n$ assez grand et d'utiliser ensuite le fait que $\mu\left[B \cap f^{-n}(B)\right]$ approche $\mu(B)^{2}$ lorsque $n$ tend vers l'infini. 


\section{Linéarisation par des homothéties}

Dans cette partie, nous montrons que la suite des itérées $\left(f^{n}\right)_{n}$ est linéarisable par des homothéties de rapport $(\sqrt{d})^{-n}$ si et seulement si $\mu$ est absolument continue par rapport à la mesure de Lebesgue. Nous adoptons la définition suivante :

Définition 2. Un système $\left(\mathbb{P}^{k}, f, \mu\right)$ de degré $d$ est dit $\sqrt{d}$-linéarisable si pour $\mu$-presque tout $x \in \mathbb{P}^{k}$, il existe $\nu(x)>0$ et une sous-suite de $\left[f^{n} \circ \tau_{x} \circ(\sqrt{d})^{-n} \operatorname{Id}_{\mathbb{C}^{\mathrm{k}}}\right]_{n}$ qui converge uniformément vers une limite injective sur $B(0, v(x))$.

Autrement dit, un système est $\sqrt{d}$-linéarisable si pour tout $x$ générique, les ellipsoïdes $\left(d_{0} f_{x}^{n}\right)^{-1}[B(0,1)]$ sont assimilables à des boules euclidiennes de rayon $(\sqrt{d})^{-n}$. Comme la taille de ces ellipsoïdes est au plus $\tau_{0}(\sqrt{d})^{-n}$ (cf théorème 3 ), il suffit d'en contrôler le volume. L'absolue continuité de $\mu$ le permet. Nous introduisons à cet effet les ensembles suivants :

$$
\left.\left.\mathcal{V}_{n}(v):=\left\{\left.x \in \mathbb{P}^{k}\left|v^{2} d^{k n} \leq\right| \operatorname{Jac} f_{x}^{n}\right|^{2} \leq \frac{1}{v^{2}} d^{k n}\right\} \quad \text { pour tout } v \in\right] 0,1\right],
$$

où Jac $f_{x}^{n}$ désigne le Jacobien complexe de $f_{x}^{n}$ en 0 . Nous obtenons le résultat suivant :

Proposition 3. Soit $\left(\mathbb{P}^{k}, f, \mu\right)$ un système de degré d. Les propriétés suivantes sont équivalentes:

1. $\mu$ est absolument continue par rapport à la mesure de Lebesgue $\omega^{k}$.

2. Les exposants du système sont tous égaux à $\log \sqrt{d}$ et il existe $\beta:] 0,1] \rightarrow \mathbb{R}^{+}$ vérifiant $\lim _{v \rightarrow 0} \beta(v)=1$ et $\liminf _{n} \mu\left[\mathcal{V}_{n}(v)\right] \geq \beta(v)$.

3. Le système est $\sqrt{d}$-linéarisable.

Nous noterons $Y$ l'ensemble $\bigcup_{p}$ Crit $f^{p}$. En tant qu'union dénombrable de sous-variétés algébriques de $\mathbb{P}^{k}$, c'est un ensemble de $\mu$-mesure nulle (voir [23]) : $\mu(Y)=0$.

Démonstration. 1) $\Rightarrow$ 2). Commençons par établir l'existence de la fonction $\beta$. On note $m=\omega^{k}$ la mesure de Lebesgue sur $\mathbb{P}^{k}$. Puisque $\mu$ est absolument continue par rapport à $m$, il existe $\varphi \in L^{1}(m)$ telle que $\mu=\varphi d m$. D'après le théorème de Lusin, il existe pour tout $n \in \mathbb{N}$ des fonctions continues $g_{n}$ et $h_{n}$ ainsi que des boréliens $C_{n}(\varphi)$ et $C_{n}\left(\varphi \circ f^{n}\right)$ vérifiant :

$$
\begin{aligned}
\varphi & =g_{n} \quad \operatorname{sur} C_{n}(\varphi) \quad \text { et } \quad \mu\left[C_{n}(\varphi)\right] \geq 1-\frac{1}{n} \\
\varphi \circ f^{n} & =h_{n} \quad \operatorname{sur} C_{n}\left(\varphi \circ f^{n}\right) \quad \text { et } \quad \mu\left[C_{n}\left(\varphi \circ f^{n}\right)\right] \geq 1-\frac{1}{n} .
\end{aligned}
$$


Soit $A_{v}:=\left\{x \in \mathbb{P}^{k} \mid v<\varphi(x)<\frac{1}{v}\right\}$ où $\left.\left.v \in\right] 0,1\right]$. On pose :

$$
Z_{n, \nu}:=\left[f^{-n}\left(A_{\nu}\right) \cap A_{\nu}\right] \cap\left[C_{n}(\varphi) \cap C_{n}\left(\varphi \circ f^{n}\right)\right] \cap Y^{c} .
$$

Rappelons que l'ensemble $Z_{n, v}^{\mathrm{Leb}}$ des points de Lebesgue de $Z_{n, v}$ est défini par :

$$
Z_{n, v}^{\mathrm{Leb}}:=\left\{x \in Z_{n, v} \mid \lim _{s \rightarrow 0} \frac{m\left[B(x, s) \cap Z_{n, v}\right]}{m[B(x, s)]}=1\right\} .
$$

L'absolue continuité de $\mu$ entraîne $\mu\left(Z_{n, v}^{\mathrm{Leb}}\right)=\mu\left(Z_{n, v}\right)$. Compte tenu du caractère mélangeant de $\mu$ et du fait que $\mu(Y)=0$, on obtient pour $n$ assez grand :

$$
\mu\left(Z_{n, v}^{\mathrm{Leb}}\right) \geq \mu\left(A_{v}\right)^{2}\left(1-\frac{v}{2}\right)-\frac{2}{n} \geq \mu\left(A_{v}\right)^{2}(1-v) .
$$

La fonction $\beta(v):=\mu\left(A_{v}\right)^{2}(1-v)$ convient si l'inclusion $Z_{n, v}^{\mathrm{Leb}} \subset \mathcal{V}_{n}(v)$ est satisfaite. Fixons donc $x \in Z_{n, v}^{\mathrm{Leb}}$. Puisque $x$ n'appartient pas à Crit $f^{n}$, il existe $s_{0}>0$ tel que $f^{n}$ soit injective sur $B\left(x, s_{0}\right)$. En outre, $x$ étant un point de Lebesgue de $Z_{n, v}$, on peut diminuer $s_{0}$ pour que $m\left[B(x, s) \cap Z_{n, v}\right] \geq \frac{1}{2} m[B(x, s)]>0$ pour tout $0<s<s_{0}$. En utilisant des changements de variables, d'abord par rapport à $\mu=\varphi d m$ qui est de Jacobien constant égal à $d^{k}$, puis par rapport à $m=\omega^{k}$, on obtient :

$$
d^{k n} \int_{B(x, s) \cap Z_{n, v}} \varphi \omega^{k}=\int_{f^{n}\left[B(x, s) \cap Z_{n, v}\right]} \varphi \omega^{k}=\int_{B(x, s) \cap Z_{n, v}} \varphi \circ f^{n}\left(f^{n *} \omega^{k}\right) .
$$

Or, puisque $C_{n}(\varphi) \cap C_{n}\left(\varphi \circ f^{n}\right)$ contient $Z_{n, \nu}$, on peut remplacer $\varphi$ par $g_{n}$ et $\varphi \circ f^{n}$ par $h_{n}$ dans ces intégrales. Après normalisation par $m(s, n, v):=m\left[B(x, s) \cap Z_{n, v}\right]$, il vient :

$$
\frac{d^{k n}}{m(s, n, v)} \int_{B(x, s) \cap Z_{n, v}} g_{n} \omega^{k}=\frac{1}{m(s, n, v)} \int_{B(x, s) \cap Z_{n, v}} h_{n}\left(f^{n *} \omega^{k}\right) .
$$

Comme les fonctions $g_{n}$ et $h_{n}$ sont continues en $x$ et $\left(f^{n *} \omega^{k}\right)_{x}=\left|\operatorname{Jac} f_{x}^{n}\right|^{2}\left(\omega^{k}\right)_{x}$, on obtient lorsque $s$ tend vers 0 :

$$
d^{k n} \varphi(x)=d^{k n} g_{n}(x)=h_{n}(x)\left|\operatorname{Jac} f_{x}^{n}\right|^{2}=\varphi \circ f^{n}(x)\left|\operatorname{Jac} f_{x}^{n}\right|^{2}
$$

c'est à dire $\frac{\left|\operatorname{Jac} f_{x}^{n}\right|^{2}}{d^{k n}}=\frac{\varphi(x)}{\varphi \circ f^{n}(x)}$. Ainsi $x \in \mathcal{V}_{n}(v)$ car $x$ et $f^{n}(x)$ appartiennent à $A_{\nu}$.

Vérifions maintenant que les exposants de $\mu$ sont minimaux. On dispose de l'égalité classique $\lim _{n} \frac{1}{n} \log \left|\mathrm{Jac} f_{x}^{n}\right|^{2}=2 \sum_{i=1}^{k} \lambda_{i}$, valable pour $\mu$-presque tout $x$ (cf par exemple [1], Section 3.3). Notons $\mathcal{V}(v):=\lim \sup _{n} \mathcal{V}_{n}(v)$ et choisissons $v$ assez petit pour que $\mu[\mathcal{V}(v)] \geq \beta(\nu) \geq \frac{1}{2}$. Comme $\lim _{n} \frac{1}{n} \log \left|\operatorname{Jac} f_{x}^{n}\right|^{2}=k \log d$ pour 
$x \in \mathcal{V}(v)$, on obtient $\sum_{i=1}^{k} \lambda_{i}=k \log \sqrt{d}$. La minimalité des exposants découle alors de la minoration $\lambda_{i} \geq \log \sqrt{d}$.

$2) \Rightarrow 3$ ). La proposition 2 s'applique car les exposants sont tous égaux à $\log \sqrt{d}$. Nous en reprenons les notations et posons

$$
\mathscr{D} V_{n}(\rho, \tau, \nu):=\mathscr{D}_{n}(\rho, \tau) \cap \mathcal{V}_{n}(v) \text { et } \mathscr{D} V(\rho, \tau, v):=\limsup _{n} \mathscr{D} V_{n}(\rho, \tau, v) \text {. }
$$

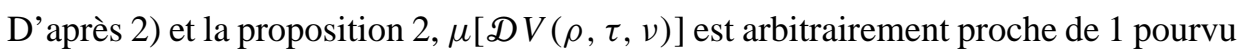
que $\rho$ et $v$ soient assez petits et $\tau$ suffisamment grand. Il suffit donc de montrer que $\left(f_{x}^{n}\right)_{n}$ est linéarisable par $\Lambda_{n}:=(\sqrt{d})^{-n} \operatorname{Id}_{\mathbb{C}^{\mathrm{k}}}$ lorsque $x \in \mathscr{D} V(\rho, \tau, \nu)$. Soit donc $\left(n_{j}\right)_{j}$ une suite strictement croissante d'entiers telle que $x \in \mathscr{D} V_{n_{j}}(\rho, \tau, \nu)$ pour tout $j$. Puisque $\mathscr{D} V_{n_{j}}(\rho, \tau, \nu) \subset \mathscr{D}_{n_{j}}(\rho, \tau) \subset \mathscr{B}_{n_{j}}(\rho)$ on a

$$
f_{x}^{n_{j}} \circ\left(d_{0} f_{x}^{n_{j}}\right)^{-1}(B(0, \rho)) \subset B\left(0, R_{0}\right) .
$$

Il s'agit donc de vérifier que $\left(d_{0} f_{x}^{n_{j}}\right)^{-1}$ est équivalente à $\Lambda_{n_{j}}$. A cet effet, notons

$$
\delta_{j, 1} \leq \cdots \leq \delta_{j, k}
$$

les valeurs singulières de $P:=\left(d_{0} f_{x}^{n_{j}}\right)^{-1}$, c'est à dire les valeurs propres de la racine carrée de $P P^{*}$, où $P^{*}$ désigne l'adjoint de $P$. Il existe en particulier deux matrices unitaires $U$ et $V$ telles que $U P V=\operatorname{Diag}\left(\delta_{j, 1}, \cdots, \delta_{j, k}\right)$. Ces valeurs singulières vérifient $\delta_{j, k} \leq \tau(\sqrt{d})^{-n_{j}}$ car $x \in \mathscr{D}_{n_{j}}(\rho, \tau)$ et $\left(\delta_{j, 1} \ldots \delta_{j, k}\right)^{2}=\left|\operatorname{Jac} f_{x}^{n_{j}}\right|^{-2} \geq$ $v^{2} d^{-k n_{j}}$ car $x \in \mathcal{V}_{n_{j}}(v)$. D'où l'on déduit les inégalités :

$$
\nu \tau^{1-k}(\sqrt{d})^{-n_{j}} \leq \delta_{j, 1} \leq \cdots \leq \delta_{j, k} \leq \tau(\sqrt{d})^{-n_{j}} .
$$

L'application $\left(d_{0} f_{x}^{n_{j}}\right)^{-1}$ est donc équivalente à l'homothétie $\Lambda_{n_{j}}$.

$3) \Rightarrow 1$ ). Soit $x \in \mathbb{P}^{k}$ un point $\mu$ générique. D'après 3$)$, il existe $\rho>0$ et une suite croissante d'entiers $\left(n_{j}\right)_{j}$ tels que $f^{n_{j}} \circ \tau_{x} \circ \Lambda_{n_{j}}: B(0, \rho) \rightarrow B\left(0, R_{0}\right)$ soit une suite d'injections. Soient $B_{r}:=\tau_{x}[B(0, r)]$ et $B_{n_{j}}:=\tau_{x}\left[B\left(0, \rho(\sqrt{d})^{-n_{j}}\right)\right]$. Il s'ensuit que :

$$
\liminf _{r \rightarrow 0} \frac{\mu\left(B_{r}\right)}{m\left(B_{r}\right)} \leq \liminf _{j} \frac{\mu\left(B_{n_{j}}\right)}{m\left(B_{n_{j}}\right)} \lesssim \liminf _{j} \frac{\mu\left(B_{n_{j}}\right)}{d^{-k n_{j}}}=\liminf _{j} \mu\left(f^{n_{j}}\left(B_{n_{j}}\right)\right) \leq 1
$$

où la dernière égalité provient du fait que $\mu$ est de jacobien constant $d^{k}$. Ceci étant vérifié pour $\mu$-presque tout $x$, la mesure $\mu$ est bien absolument continue par rapport à $m$ (cf [20], Theorem 2.12).

Remarque 2. Comme nous l'avons fait pour établir le théorème 3, une légère modification dans la preuve de 2) $\Rightarrow 3$ ) permet de choisir la sous-suite $\left(n_{j}\right)_{j}$ de façon à ce que $f^{n_{j}}(x)$ ne s'échappe pas d'un borélien $B$ de $\mu$-mesure strictement positive prescrit. 


\section{Régularisation du courant de Green}

Nous achevons ici la preuve du théorème 1 . D'après la proposition 3 , il s'agit de caractériser les systèmes $\left(\mathbb{P}^{k}, f, \mu\right)$ qui sont $\sqrt{d}$-linéarisables. La démonstration repose sur le lemme 3 ci-dessous. Commençons par introduire quelques définitions. On notera $S:=S_{a}+S_{s}$ la décomposition de Lebesgue d'un $(1,1)$-courant positif $S$. Celle-ci peut être définie à partir de la décomposition de Lebesgue des mesures car un tel courant peut être considéré comme une $(1,1)$-forme à coefficients mesures. Il est très facile de voir que cette décomposition est unique et que les courants $S_{a}, S_{s}$ restent positifs. Par contre la fermeture éventuelle de $S$ n'implique pas celle de $S_{a}$ ou de $S_{s}$. Nous noterons $\operatorname{Supp}(S)$ le support de $S$ et $\sigma_{S}:=S \wedge \omega^{k-1}$ sa mesure trace. On voit facilement que la décomposition de Lebesgue de $\sigma_{S}$ est donnée par $\sigma_{S}=\sigma_{S_{a}}+\sigma_{S_{S}}$.

Lemme 3. Soient $\left(\mathbb{P}^{k}, f, \mu\right)$ un système $\sqrt{d}$-linéarisable, $S$ un courant positif de bidegré $(1,1)$ sur $\mathbb{P}^{k}$ tel que $f^{*} S=d S$ ( $S$ n'est pas nécessairement fermé) et $\Omega$ un ouvert de $\mathbb{P}^{k}$ chargé par $\mu$.

1) Si S est absolument continu sur $\Omega\left(S=S_{a}\right)$ alors il existe une boule $B(0, r) \subset \mathbb{C}^{k}$, un ouvert $\Omega^{\prime} \subset \Omega$ chargé par $\mu$ et un biholomorphisme $\Phi: B(0, r) \rightarrow \Omega^{\prime} \subset \Omega$ tels que $\Phi^{*} S$ soit une forme différentielle à coefficients constants sur $B(0, r)$.

2) Supposons que $S$ dérive d'un potentiel psh continu $v$ sur $\Omega\left(S=d d^{c} v\right)$. Si $S_{a}$ est nul sur $\Omega$ alors $\mu(\Omega \cap \operatorname{Supp} S)=0$.

Démonstration du théorème 1 . Soit $\Omega$ un ouvert de $\mathbb{P}^{k}$ chargé par $\mu$. La première assertion du lemme 3 appliquée à $T_{a}$ permet de supposer que dans de bonnes coordonnées, la restriction de $T_{a}$ à $\Omega$ est donnée par une forme $H$ à coefficients constants. En particulier $T_{a}$ possède un potentiel continu sur $\Omega$ et il en va donc de même pour $T_{s}=T-T_{a}$ car $T$ est à potentiels locaux continus. Ceci permet, sur $\Omega$, d'exprimer $\mu$ sous la forme d'une somme de mesures positives obtenues comme produits extérieurs de $T_{a}$ et $T_{s}$ :

$$
\mu=T^{k}=\left(T_{a}+T_{s}\right)^{k}=T_{a}^{k}+\sum_{j=1}^{k} C_{k}^{j} T_{s}^{j} \wedge T_{a}^{k-j} .
$$

Puisque $\left(T_{s}\right)_{a}$ est identiquement nul par définition, la seconde assertion du lemme 3 montre que $\mu$ ne charge pas $\Omega \cap \operatorname{Supp} T_{s}$ et donc, au vu de (7), la mesure $T_{a}^{k}$ n'est pas identiquement nulle sur $\Omega$. Autrement dit la forme $H$ n'est pas dégénérée. Par ailleurs, puisque $\mu$ est absolument continue, chaque terme du second membre de (7) doit, en tant que mesure positive, être absolument continue. En particulier, la mesure singulière $T_{s} \wedge T_{a}^{k-1}$ est nulle sur $\Omega$. Or, $H$ étant strictement positive, celle-ci est équivalente à la mesure trace $\sigma_{T_{s}}$ de $T_{s}$. Le courant (positif) $T_{s}$ est donc nul sur $\Omega$ et $T$ coïncide sur cet ouvert avec une forme lisse définie positive. L'endomorphisme $f$ 
est donc un exemple de Lattès comme cela est démontré dans [2] (voir le résultat cité dans l'introduction).

Démonstration du lemme 3. Pour simplifier les notations, nous ne ferons pas figurer les cartes locales $\tau_{x}$ dans cette démonstration. Nous notons $\Lambda_{n}$ l'homothétie $(\sqrt{d})^{-n} \operatorname{Id}_{\mathbb{C}^{\mathrm{k}}}$.

1) Puisque $S$ est absolument continu sur $\Omega$, il est de la forme

$$
S=\frac{i}{2} \sum_{1 \leq p, q \leq k} h_{p, q}(z) d z_{p} \wedge d \bar{z}_{q} \quad \text { où } h_{p, q} \in L^{1}(\Omega) .
$$

Soit $\mathcal{M}$ l'ensemble des points de $\Omega$ où toutes les fonctions $h_{p, q}$ sont continues en moyenne, c'est à dire :

$$
\lim _{r \rightarrow 0} \frac{1}{m(B(z, r))} \int_{B(z, r)} h_{p, q}(t) d m(t)=h_{p, q}(z) \quad \text { pour tout } z \in \mathcal{M} .
$$

Puisque le système est $\sqrt{d}$-linéarisable, $\mu$ est absolument continue par rapport à $m$ et l'ensemble $\mathcal{M}$ est de mesure totale pour $m$ et $\mu$. Notons $\mathcal{R}$ l'ensemble des points de $\Omega \cap \operatorname{Supp} \mu$ où la suite $\left(f^{n}\right)_{n}$ est linéarisable par des homothéties de rapport $(\sqrt{d})^{-n}$. Comme $\mu$ est absolument continue, la proposition 3 nous assure que $\mu(\mathcal{M} \cap \mathcal{R})>0$. Soit alors $z \in \mathcal{M} \cap \mathcal{R}$ et posons $\Phi_{n}:=f^{n} \circ \Lambda_{n}$ (on identifie $z$ avec l'origine de $\mathbb{C}^{k}$ ). Quitte à prendre une sous-suite, $\Phi_{n}(0)=f^{n}(z)$ reste dans $V \cap \operatorname{Supp} \mu$ où $V$ est un voisinage de $z$ (cf Remarque 2) et la suite $\left(\Phi_{n}\right)_{n}$ converge vers un biholomorphisme $\Phi: B(0, v) \rightarrow \Omega^{\prime} \subset \Omega$. Le support de $\mu$ étant fermé et invariant, on a $\Phi(0) \in \Omega \cap \operatorname{Supp} \mu$ et donc $\mu\left(\Omega^{\prime}\right)>0$. L'invariance de $S$ entraîne :

$$
\Phi_{n}^{*} S=\Lambda_{n}^{*} f^{n *} S=d^{n} \Lambda_{n}^{*} S=\frac{i}{2} \sum_{1 \leq p, q \leq k} h_{p, q} \circ \Lambda_{n} d z_{p} \wedge d \bar{z}_{q} .
$$

Puisque $z \in \mathcal{M}$, on obtient $\Phi^{*} S=\frac{i}{2} \sum_{1 \leq p, q \leq k} h_{p, q}(0) d z_{p} \wedge d \bar{z}_{q}$ par passage à la limite.

2) Supposons $\mu(\Omega \cap \operatorname{Supp} S)>0$ et montrons que $S_{a}$ est non nul. Quitte à diminuer $\Omega$ on peut supposer que $S=d d^{c} v$ sur un voisinage $\tilde{\Omega}$ de $\bar{\Omega}$. Quitte à choisir une carte locale, $\tilde{\Omega}$ est un ouvert de $\mathbb{C}^{k}$. D'après la proposition 3 , il existe $\mathcal{R} \subset \Omega \cap \operatorname{Supp} S$ de $\mu$-mesure positive tel que pour tout point $z \in \mathcal{R}$, il existe une sous-suite $\Phi_{n_{j}}:=f^{n_{j}} \circ\left(z+\Lambda_{n_{j}}\right)$ convergeant uniformément sur $B(0, v(z))$ vers un biholomorphisme $\Phi$. On peut aussi supposer que $f^{n_{j}}(z) \in \mathcal{R}$ (cf remarque 2).

Observons tout d'abord qu'il suffit de montrer que $\sigma_{S}$ possède une dérivée de Radon-Nykodym strictement positive en tout point $z$ de $\mathcal{R}$ :

$$
\lim _{n} \frac{1}{d^{-k n}} \int_{B\left(z, v(\sqrt{d})^{-n}\right)} S \wedge \omega_{0}^{k-1}>0 \quad \text { pour tout } z \in \mathcal{R},
$$


où $\omega_{0}$ désigne la forme standard $\frac{i}{2} d d^{c}\|z\|^{2}$. En effet, comme $\mu(\mathcal{R})>0$, cette propriété montre que la mesure $\sigma_{S_{a}}$ (qui est égale à $\left.\left(\sigma_{S}\right)_{a}\right)$ n'est pas triviale sur $\Omega$ et il s'ensuit que le courant positif $S_{a}$ n'est pas nul.

Vérifions à présent la stricte positivité des dérivées. Notons que quitte à supprimer à $\mathcal{R}$ un ensemble de mesure de Lebesgue nulle (donc de $\mu$-mesure nulle), ces dérivées existent en tout point de $\mathcal{R}$. Fixons donc $z \in \mathcal{R}$, et reprenons les applications $\Phi_{n_{j}}$ et $\Phi$ précédentes. Comme $\Phi(0) \in \bar{\Omega}$ on peut diminuer $v$ de façon à ce que les ouverts $\Phi_{n_{j}}(B(0, v))$ et $\Phi(B(0, v))$ soient contenus dans $\bar{\Omega}$. Puisque $f^{*} S=d S$, il vient :

$$
\begin{aligned}
\frac{1}{d^{-k n_{j}}} \int_{B\left(z, v(\sqrt{d})^{-n_{j}}\right)} S \wedge \omega_{0}^{k-1} & =\frac{1}{d^{-(k-1) n_{j}}} \int_{z+\Lambda_{n_{j}}[B(v)]} f^{n_{j} *} S \wedge \omega_{0}^{k-1} \\
& =\frac{1}{d^{-(k-1) n_{j}}} \int_{B(v)} \Phi_{n_{j}}^{*} S \wedge\left(\Lambda_{n_{j}}^{*} \omega_{0}\right)^{k-1} \\
& =\int_{B(v)} \Phi_{n_{j}}^{*} S \wedge \omega_{0}^{k-1} \\
& =\int_{B(v)} d d^{c}\left(v \circ \Phi_{n_{j}}\right) \wedge \omega_{0}^{k-1},
\end{aligned}
$$

où $B(r)$ désigne la boule centrée en l'origine et de rayon $r$. Le théorème de convergence dominée entraîne alors :

$\lim _{j} \frac{1}{d^{-k n_{j}}} \int_{\left.B(v \sqrt{d})^{-n_{j}}\right)} S \wedge \omega_{0}^{k-1} \geq \int_{B(v)} d d^{c}(v \circ \Phi) \wedge \omega_{0}^{k-1}=\int_{B(v)} \Phi^{*} S \wedge \omega_{0}^{k-1}$.

Cette dernière intégrale est bien strictement positive, car $\Phi(0) \in \overline{\mathcal{R}} \subset \operatorname{Supp} S$.

\section{Appendice}

Nous résumons ici la preuve du théorème du pluripotentiel utilisé dans la section 4, ainsi que celle de l'estimation de la mesure présentée dans l'introduction.

7.1. Un théorème de la théorie du pluripotentiel. Il s'agit d'établir la version suivante d'un résultat dû à Briend-Duval [5] :

Théorème. Soit $S:=d d^{c} w$ un $(1,1)$ courant positif fermé de potentiel $w$ continu sur le polydisque $P(0, R)$ et $E \subset P\left(0, \frac{R}{2}\right)$. On suppose que par tout $z \in E$ passe un disque holomorphe $\sigma_{z}: \Delta \rightarrow \mathbb{C}^{k}$ de taille l et qu'il existe une fonction $h_{z}$ harmonique sur $\Delta$ telle que $\left|w \circ \sigma_{z}-h_{z}\right| \leq \epsilon$ sur $\Delta$. Alors il existe une constante $C(w)$ ne dépendant que de $w$ telle que $S^{k}(E) \leq C(w) \frac{k^{2}}{l^{2}} \epsilon$. 
Rappelons qu'un disque holomorphe $\sigma: \Delta \rightarrow \mathbb{C}^{k}$ passant par $z \in \mathbb{C}^{k}$ est dit de taille $l>0$ si il est de la forme $\sigma(u)=z+l u . v+\beta(u)$ où $v$ est un vecteur unitaire de $\mathbb{C}^{k}, \beta(0)=0$ et $\|\beta\| \leq \frac{l}{1000}$.

Démonstration. Soit $p_{l}$ la projection sur le $l$-ième axe de $\mathbb{C}^{k}$ et $E_{l}:=\{z \in E \mid$ $\left.\left\|p_{l}\left(v_{z}\right)\right\| \geq \frac{1}{\sqrt{k}}\right\}$, de sorte que $E=\bigcup_{l=1, k} E_{l}$. Pour fixer les idées nous allons estimer $S^{k}\left(E_{1}\right)$. A cet effet, on recouvre le polydisque $P\left(0, \frac{1}{2} R\right)$ par environ $N:=$ $\frac{1}{4} \frac{100 k}{l^{2}}$ ellipsoïdes contenus dans $P(0, R)$ de la forme $\mathcal{E}[B(0, R)]$ où $\mathcal{E}\left(z_{1}, z^{\prime}\right)=$ $\left(\frac{l}{10 \sqrt{k}} z_{1}, z^{\prime}\right)$.

Soit $\mathscr{E}$ l'un de ces ellipsoïdes. Puisque $\mathscr{E}$ est strictement pseudoconvexe, il existe une fonction $\hat{w}$ p.s.h maximale sur $\mathcal{E}$, continue sur $\bar{\varepsilon}$ et coïncidant avec $w$ sur $b \mathcal{E}$. Si $z \in \mathcal{E} \cap E_{1}$, on voit facilement que le disque $\sigma_{z}(\Delta)$ traverse $\mathcal{E}$, au sens où la composante connexe $\mathcal{C}$ de $\sigma_{z}^{-1}\left(\mathcal{E} \cap \sigma_{z}(\Delta)\right)$ contenant l'origine est relativement compacte dans $\Delta$. Un argument de principe du maximum montre que $\mathcal{C}$ est simplement connexe. En l'exhaustant par des domaines à bord suffisamment régulier, on peut paramétrer des disques holomorphes contenus dans $\mathcal{E}$ et dont le bord est arbitrairement proche de $b \&$. Plus précisément, $\epsilon>0$ étant fixé, on trouve une transformation conforme et continue jusqu'au bord $\psi: \bar{\Delta} \rightarrow \psi(\bar{\Delta}) \subset \mathcal{E}$ telle que $\psi(0)=0$ et $|\hat{w}-w| \leq \epsilon$ sur $\sigma_{z} \circ \psi(b \Delta)$. Posons $\tilde{\sigma}_{z}:=\sigma_{z} \circ \psi$ et notons $\tilde{h}$ la fonction harmonique sur $\Delta$ continue sur $\bar{\Delta}$ et coïncidant avec $w \circ \tilde{\sigma}_{z}$ sur $b \Delta$. On a alors :

$$
w(z) \leq \hat{w}(z) \leq \tilde{h}(0)+\epsilon
$$

la première inégalité provient de la maximalité de $\hat{w}$ sur $\mathscr{E}$ et la seconde du principe du maximum appliqué à $\hat{w} \circ \tilde{\sigma}_{z}-\tilde{h}$ (cette fonction coïncide avec $\hat{w} \circ \tilde{\sigma}_{z}-w \circ \tilde{\sigma}_{z} \operatorname{sur} b \Delta$ ).

Par hypothèse on a $h_{z} \circ \psi-\epsilon \leq w \circ \sigma_{z} \circ \psi=w \circ \tilde{\sigma}_{z} \leq h_{z} \circ \psi+\epsilon$ sur $\bar{\Delta}$. On a donc aussi $h_{z} \circ \psi-\epsilon \leq \tilde{h} \leq h_{z} \circ \psi+\epsilon$ et il s'ensuit que :

$$
|w(z)-\tilde{h}(0)| \leq 2 \epsilon .
$$

Les inégalités (8) et (9) montrent que :

$$
\mathcal{E} \cap E_{1} \subset \mathcal{E}(w, \epsilon):=\{z \in \mathcal{E} \mid 0 \leq \hat{w}(z)-w(z) \leq 3 \epsilon\} .
$$

La majoration annoncée résulte alors immédiatement de l'estimation suivante qui est au coeur de la démonstration de Briend-Duval et pour laquelle nous renvoyons à [5] ou [23] page 180, Théorème A.10.2 :

Il existe une constante $C(w)>0$ telle que $\left(d d^{c} w\right)^{k}[\mathcal{E}(w, \epsilon)] \leq C(w) \epsilon$.

7.2. Estimation de la dimension. Nous esquissons la preuve de l'estimation de la dimension en reprenant mutatis mutandis les arguments développés par Binder et DeMarco [4] dans le cas des endomorphismes polynomiaux de $\mathbb{C}^{k}$. 
Théorème. Soit un système $\left(\mathbb{P}^{k}, f, \mu\right)$ de degré $d$ et d'exposants $\lambda_{1} \leq \cdots \leq \lambda_{k}$. La dimension de $\mu$ vérifie $: \operatorname{dim}(\mu) \leq 2(k-1)+\frac{\log d}{\lambda_{k}}$.

Rappelons que la dimension est définie comme la borne inférieure des dimensions de Hausdorff des boréliens de mesure totale. Ce résultat montre que si la dimension de $\mu$ est égale à $2 k$, alors tous les exposants de $\mu$ sont minimaux, égaux à $\log \sqrt{d}$.

Démonstration. Il s'agit d'exhiber pour tout $\epsilon>0$ un borélien $Y$ de mesure totale vérifiant :

$$
\operatorname{dim}_{H}(Y) \leq 2(k-1)+\frac{\log d}{\lambda_{k}}+\frac{2 k}{\lambda_{k}} \epsilon .
$$

Soit $\widehat{A}$ l'ensemble des points $\hat{x}=\left(x_{n}\right)_{n \geq 0}$ de $\widehat{\mathbb{P}^{k}}$ vérifiant pour tout $n \geq 0$ :

$$
B\left(x_{-n}, \frac{r_{0}}{\kappa_{0}} e^{-n\left(\lambda_{k}+\epsilon\right)}\right) \subset f_{\hat{x}}^{-n}\left[B\left(x_{0}, r_{0}\right)\right]
$$

et

$$
m\left(f_{\hat{x}}^{-n}\left[B\left(x_{0}, r_{0}\right)\right]\right) \leq \kappa_{0} e^{-2 n\left(\lambda_{1}+\cdots+\lambda_{k}\right)+n \epsilon} .
$$

On rappelle que $m$ désigne la mesure volume standard sur $\mathbb{P}^{k}$. On vérifie que si $\kappa_{0}$ est assez grand et $r_{0}$ assez petit, alors $\hat{\mu}(\widehat{A})>0$ (cf [4], lemme 2).

Soit $\widehat{A_{n}}:=\hat{f}^{-n} \widehat{A}$. La mesure $\hat{\mu}$ étant ergodique, le théorème de Birkhoff entraîne que $\widehat{Y}:=\lim \sup _{n} \widehat{A_{n}}$ est de mesure totale.

On pose alors $Y:=\pi_{0}(\widehat{Y})$ et $A_{n}:=\pi_{0}\left(\widehat{A_{n}}\right)$, de sorte que $Y$ est aussi de mesure totale et est contenu dans $\lim \sup _{n} A_{n}$. Estimer la dimension de Hausdorff de $Y$ revient à estimer celle des ensembles $A_{n}$, pour $n$ assez grand. Par définition de $\widehat{A}$, tout point $y$ de $A_{n}$ vérifie :

(1) $f^{n}$ admet une branche inverse $g_{n}$ sur $B\left(f^{n}(y), r_{0}\right)$, telle que $g_{n}\left(f^{n}(y)\right)=y$

(2) La boule $B\left(y, \frac{r_{0}}{\kappa_{0}} e^{-n\left(\lambda_{k}+\epsilon\right)}\right)$ contient $\mathcal{P}:=g_{n}\left[B\left(f^{n}(y), r_{0}\right)\right]$

(3) $m(\mathcal{P}) \leq k_{0} e^{-2 n\left(\lambda_{1}+\cdots+\lambda_{k}\right)+n \epsilon}$.

Il découle de ces propriétés que $A_{n}$ est recouvert par une famille $\left(\mathcal{P}_{i}\right)_{i \in I}$ d'ouverts du type $\mathcal{P}$ dont le cardinal est de l'ordre de $d^{k n}$. Pour le voir, il suffit de recouvrir $\overline{A_{0}}$ par un nombre fini de boules $B\left(x_{i_{0}}, \frac{1}{4} r_{0}\right)$ puis d'observer que tout $y \in A_{n}$ est dans $g_{n}\left[B\left(x_{i_{0}}, \frac{1}{2} r_{0}\right)\right]$ dès lors que $f^{n}(y) \in B\left(x_{i_{0}}, \frac{1}{4} r_{0}\right)$. D'après le point 3 , le volume de la réunion des $\mathcal{P}_{i}$ n'excède pas $d^{k n} e^{-2 n\left(\lambda_{1}+\cdots+\lambda_{k}\right)+n \epsilon}$.

Considérons à présent un recouvrement $\left(\mathcal{M}_{j}\right)_{j \in J}$ de $A_{n}$ par des sous-ensembles de diamètre $\frac{r_{0}}{100 \kappa_{0}} e^{-n\left(\lambda_{k}+\epsilon\right)}$ provenant d'un maillage de $\mathbb{P}^{k}$. D'après le point 2 , un sous-ensemble $\mathcal{M}_{j}$ intersectant $A_{n}$ est nécessairement contenu dans $\bigcup_{i \in I} \mathcal{P}_{i}$. On a donc :

$$
\operatorname{Card}(J) \leq \frac{m\left(\bigcup_{i \in I} \mathcal{P}_{i}\right)}{m\left(\mathcal{M}_{j}\right)} \lesssim \frac{d^{k n} e^{-2 n\left(\lambda_{1}+\cdots+\lambda_{k}\right)+n \epsilon}}{\left(e^{-n\left(\lambda_{k}+\epsilon\right)}\right)^{2 k}}
$$


En minorant les exposants $\lambda_{1}, \ldots, \lambda_{k-1}$ par $\log \sqrt{d}$, on obtient :

$$
\operatorname{Card}(J) \lesssim d^{n} e^{n\left(\left[2(k-1) \lambda_{k}\right]+(2 k+1) \epsilon\right)} .
$$

Il s'ensuit que la mesure de Hausdorff de $A_{n}$, de dimension $l_{\epsilon}=2(k-1)+\log d / \lambda_{k}+$ $2 k \epsilon / \lambda_{k}$, est minorée par $e^{-n \epsilon}$ pour $n$ assez grand. La $l_{\epsilon}$-mesure de Hausdorff de $Y \subset \lim \sup _{n} A_{n}$ est donc finie pour tout $\epsilon>0$. Cela termine la démonstration, car $Y$ est un borélien de mesure totale.

\section{Références}

[1] L. Arnold, Random Dynamical Systems. Monogr. Math., Springer-Verlag, Berlin1998. Zbl 0906.34001 MR 1723992

[2] F. Berteloot, J. J. Loeb, Une caractérisation géométrique des exemples de Lattès de $\mathbb{P}^{k}$. Bull. Soc. Math. Fr. 129 (2) (2001), 175-188. Zbl 0994.32026 MR 1871293

[3] F. Berteloot, V. Mayer, Rudiments de dynamique holomorphe. Cours Spécialisés 7, SMF et EDP Sciences, Paris 2001. Zbl 1051.37019 MR 1973050

[4] I. Binder, L. DeMarco, Dimension of pluriharmonic measure and polynomial endomorphisms of $\mathbb{C}^{n}$. Internat. Math. Res. Not. 11 (2003), 613-625.Zbl 1027.37027 MR 1949354

[5] J. Y. Briend, J. Duval, Exposants de Liapounoff et distribution des points périodiques d'un endomorphisme de $\mathbb{P}^{k}$. Acta Math. 182 (2) (1999), 143-157. Zbl 01541209 MR 1710180

[6] J. Y. Briend, J. Duval, Deux caractérisations de la mesure d'équilibre d'un endomorphisme de $\mathbb{P}^{k}$. Inst. Hautes Études Sci. Publ. Math. 93 (2001), 145-159. Zbl 1010.37004 MR 1863737

[7] T. C. Dinh, C. Dupont, Dimension de la mesure d'équilibre d'applications méromorphes. J. Geom. Anal., à paraître.

[8] T. C. Dinh, N. Sibony, Sur les endomorphismes holomorphes permutables de $\mathbb{P}^{k}$. Math. Ann. 324 (1) (2002), 33-70. Zbl 01837353 MR 1931758

[9] C. Dupont, Propriétés extrémales et caractéristiques des exemples de Lattès, Thèse de doctorat de l'Université Paul Sabatier, Toulouse 2002.

[10] C. Dupont, Exemples de Lattès et domaines faiblement sphériques de $\mathbb{C}^{n}$. Manuscripta Math. 111 (3) (2003), 357-378. Zbl 1041.37020 MR 1993500

[11] C. Dupont, Formule de Pesin et applications méromorphes. Soumis.

[12] J. E. Fornaess, N. Sibony, Complex dynamics in higher dimensions. in Complex potential theory (Montréal, PQ, 1993), NATO ASI Ser. Math. Phys. Sci. 439, Kluwer Academic Publishers, Dordrecht 1994, 131-186. Zbl 0811.32019 MR 1332961

[13] J. E. Fornaess, N. Sibony, Complex dynamics in higher dimensions II. In Modern methods in complex analysis (Princeton, NJ, 1992), Ann. of Math. Stud. 137, Princeton University Press, Princeton, NJ, 1995, 135-182. Zbl 0847.58059 MR 1369137

[14] J. E. Fornaess, N. Sibony, Some open problems in higher dimensional complex analysis and complex dynamics. Publ. Mat. 45 (2) (2001), 529-547. Zbl 0993.32001 MR 1876919 
[15] J. H. Hubbard, P. Papadopol, Superattractive fixed points in $\mathbb{C}^{n}$. Indiana Univ. Math. J. 43 (1) (1994), 321-365. Zbl 0858.32023 MR 1275463

[16] M. Jonsson, D. Varolin, Stable manifolds of holomorphic diffeomorphisms. Invent. Math. 149 (2) (2002), 409-430. Zbl 1048.37047 MR 1918677

[17] F. Ledrappier, Some properties of absolutely continuous invariant measure on an interval. Ergodic Theory Dynamical Systems 1 (1) (1981), 77-93. Zbl 0487.28015 MR 0627788

[18] F. Ledrappier, Quelques propriétés ergodiques des applications rationnelles. C. R. Acad. Sci. Paris Sér. I Math. 299 (1) (1984), 37-40. Zbl 0567.58016 MR 0756305

[19] C. T. McMullen, Frontiers in complex dynamics. Bull. Amer. Math. Soc. (N.S.) 31 (2) (1994), 155-172. Zbl 0807.30013 MR 1260523

[20] P. Mattila, Geometry of sets and measures in euclidian spaces. Cambridge Stud. Adv. Math. 44, Cambridge University Press, Cambridge 1995. Zbl 0819.28004 MR 1333890

[21] V. Mayer, Comparing measures and invariant line fields. Ergodic Theory Dynamical Systems 22 (2) (2002), 555-570. Zbl 01779087 MR 1898805

[22] J. Milnor, On Lattès Maps. Stony Brook IMS Preprint, 2004, http ://www.math.sunysb.edu/ $\sim$ jack/

[23] N. Sibony, Dynamique des applications rationnelles de $\mathbb{P}^{k}$. In Dynamique et Géométrie Complexes, Panoramas et Synthèses 8, SMF et EDP Sciences, Paris 1999. Zbl 1020.37026 MR 1760844

[24] A. Zdunik, Parabolic orbifolds and the dimension of the maximal measure for rational maps. Invent. Math. 99 (3) (1990), 627-649. Zbl 0820.58038 MR 1032883

Received May 5, 2003; revised July 10, 2004

F. Berteloot, Université P. Sabatier, Toulouse III, Lab. Emile Picard, Bat. 1R2, UMR 5580, 118, route de Narbonne, 31062 Toulouse Cedex, France

E-mail: berteloo@picard.ups-tlse.fr

C. Dupont, Université Paris-Sud, Mathématique, Bat. 425, UMR 8628, 91405 Orsay, France

E-mail: christophe.dupont@math.u-psud.fr 